\title{
Methods of Channel Estimation and Symbol Detection for IEEE 802.15.4 Considering the Wireless Channel in the High Speed Train
}

\author{
Jingu Lee, Minho Kim, Daehyun Kim, Jonghwa Lee, Jungyeol Kim, and Younglok Kim
}

\begin{abstract}
The monitoring system in railroad system is under study to prevent any derailment and accident caused by any defect in train. Since the monitoring system is composed of wireless sensor network based on IEEE 802.15.4 in train environment, we need to analyze the wireless channel of special case like the train environment which is very tough for wireless communication by power loss, multipath and fast fading. After confirmation of the estimated channel information from the measured signal in train environment, we proposed channel estimation method considering estimated channel information. The main advantage of the proposed channel estimation method using characteristic of chip code is possible to simply when channel has significant 2-tap like train environment. Also, since the IEEE 802.15.4 system is not specified about the channel and equalization, we proposed the symbol detection method having equalization for the multipath fading. We simulate a bit error probability performance to verify simple method without performance degradation.
\end{abstract}

Index Terms-Fast fading, railroad, channel estimation, IEEE 802.15.4, sensor network, equalization.

\section{INTRODUCTION}

Korea Railroad Research Institute(KRRI) has researched on how to monitor the state of a train or railroad on a real time basis by installing vibration sensors on the outside of the train and organizing it to a sensor network in order to prevent any derailment and accident caused by any defect in the train. Present monitoring systems in railroad systems almost exclusively use wired sensor networks, and recently, the demand for monitoring systems with wireless sensor networks has expanded in accordance with their easy installation in places that had previously been considered difficult to access and install in. However, the transmitter responsible for sending the data from the sensor has a limited power supply as it is installed outside of the train. Therefore, the self-power generation of 'Energy Harvesting' has been adopted for the power supply because it does not require the transformation of the structure of the conventional train. But, due to the limited power from the energy harvesting, the study is required to operate the wireless sensor network system in low power [1], [2]. So, Korea Railroad Research Institute(KRRI) and Electronics and Telecommunication Research Institute(ETRI) selected the IEEE802.15.4 system as the most

Manuscript received September 10, 2014; revised June 3, 2015

The authors are with the Electrical Engineering Department, University of Sogang, Seoul, 121-742, Korea (e-mail: ylkim@sogang.ac.kr, jingu8562@sogang.ac.kr, minho89@sogang.ac.kr, sinzuggo@sogang.ac.kr kjr5594@ sogang.ac.kr, jhlee1124@sogang.ac.kr). suitable standard for organizing the sensor's network in the train environment because it has advantages in the low power wireless sensor network for the operation of the system in low power.

As IEEE 802.15.4 is applied to the special environment of high speed train which runs in high speed and which is made with metals, it is necessary to check the wireless channel in the actual high speed train environment. Especially, as the multipath delay component causes the inter-symbol interference (ISI) and thereby the degradation of performance, the analysis of the multipath delay component is required. In addition, once the multipath component is understood, the equalization algorithm is required to reduce the performance of degradation by ISI. But until now the IEEE 802.15.4 standard has no specification of the equalization method [3].

Accordingly, in this paper, the components of the wireless channel using the measured signal at the inside from transmitted signal at the outside of the train would be analyzed based on IEEE 802.15.4 and the simple wireless channel estimation algorithm and the equalization algorithm according to the characteristics of the wireless channel component would be proposed.

This paper is composed as follows. Chapter 2 describes the characteristics of the train environment and the technological features of IEEE 802.15.4 PHY (physical layer). Chapter 3 refers to the estimation of the carrier frequency offset(CFO) which is caused by the unconformity between oscillators of senders and receivers, And refers to the analysis of the measuring signal based on the IEEE 802.15.4 system of the communication between inside and outside of the high speed train and describes the channel analysis through the least square(LS) channel estimation method. Chapter 4 proposes the channel estimation method and the symbol detection method of a simple channel, based on the profile from the analysis result of the wireless channel of a high speed train in Chapter 3. Chapter 5 shows the excellence of proposed methods through the performance evaluation simulation. Chapter 6 contains conclusions.

\section{TRAIN ENVIRONMENT AND IEEE 802.15.4 SYSTEM}

\section{A. What Is the Train Environment?}

The train environment has a lot of physical obstruction factors as far as the wireless sensor network is organized. The train is usually made of metals and moves under severe vibration and in high speed causing a lot of multipath fading, noise from vibration, and fast fading from high movement. All 
these factors cause the degradation of all receiving performances.

\section{B. Organization of the Sensor Network}

The sensors are attached on the outside of the train (Fig. 1) and are supplied with self-generated power and then transmit the data to the inside of the train. At this time, the wireless communication between the inside and outside of the train is done using the IEEE 802.15.4 communication system. As the receiver gets the power from the train, it has no limitation in power supply. Then, it compensates and equalizes the received signal and then transmits the data to the server. ETRI made an experiment on the basis of the above-mentioned. And we analyzed the data measured in this experiment.

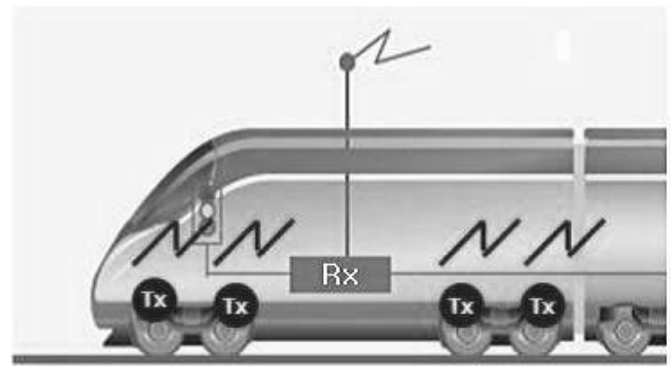

Fig. 1. Sensor network on train

\section{IEEE 802.15.4 Standard}

IEEE 802.15.4 technology uses two bands of 868/915 MHz and $2.45 \mathrm{GHz}$. The band of $868 / 915 \mathrm{MHz}$ uses the BPSK modulated DSSS (Direct sequence spread spectrum) while the band of $2.45 \mathrm{GHz}$ uses the OQPSK modulated 16-ary quasi-orthogonal DSSS method. The parameters according to the used frequency bands are specified in Table I. In this paper, they are based on the IEEE 802.15.4 LR-WPAN system of the frequency band of $2.45 \mathrm{GHz}$ which is the standard for Korea Railroad Research Institute (KRRI).

TABLE I: PARAMETERS ACCORDING TO THE USED FREQUENCY BANDS

\begin{tabular}{|c|c|c|c|c|c|c|}
\hline \multirow{2}{*}{$\begin{array}{l}\text { PHY } \\
(\mathrm{MHz})\end{array}$} & \multirow{2}{*}{$\begin{array}{c}\text { Frequency } \\
\text { band } \\
(\mathrm{MHz})\end{array}$} & \multicolumn{3}{|c|}{ Spreading Parameters } & \multicolumn{2}{|c|}{ Data parameters } \\
\hline & & $\begin{array}{l}\text { Chip rate } \\
\text { (kchip/s) }\end{array}$ & Modulation & $\begin{array}{c}\text { Bit } \\
\text { rate } \\
(\mathrm{kb} / \mathrm{s}) \\
\end{array}$ & $\begin{array}{l}\text { Symbol rate } \\
\text { (ksymbols/s) }\end{array}$ & Symbols \\
\hline \multirow{2}{*}{$868 / 915$} & $868-868.6$ & 300 & BPSK & 20 & 20 & Binary \\
\hline & $902-928$ & 600 & BPSK & 40 & 40 & Binary \\
\hline 2450 & $\begin{array}{c}2400 \\
-2483.5\end{array}$ & 2000 & O-QPSK & 250 & 62.5 & $\begin{array}{c}\text { 16-ary } \\
\text { Orthogonal }\end{array}$ \\
\hline
\end{tabular}

2.45 GHz PHY of IEEE 802.15.4 system uses the industrial scientific medical (ISM) band now. It adopts the O-QPSK modulation method which delays the Q-channel by $T_{c}$, compared to the I-channel so that it can be resistant to the interference of nearby networks and systems.

There are four kinds of packets such as beacon packet, data packet, acknowledge packet and MAC command packet according to the purpose of usage. In this paper the most widely used data packet has been adopted to perform the analysis of performance. PHAY protocol data unit (PPDU) is composed of the preamble 4bytes for the synchronization, the synchronization header (SHR) composed of start of packet delimiter (SFD) 1 byte, PHY header (PHY) and PHY service data unit (PSDU) and the size of PSDU could be set in a range of $0 \sim 127$ bytes. In this paper, the size of the total packets is 50 bytes.

The 16-ary quasi-orthogonal modulation method is used in IEEE 802.15.4 so that the column of information bits of $250 \mathrm{~kb} / \mathrm{s}$ generated on the top layer is symbol mapped in the unit of 4 bits and that each mapped symbol spreads into the chip sequence as proposed in Table II.

TABLE II: CHIP SEQUENCE MAPPED FOR EACH SYMBOL

\begin{tabular}{|c|c|c|}
\hline \multicolumn{2}{|c|}{ Symbol } & Chip values \\
\hline $\begin{array}{c}\text { Decimal } \\
(i)\end{array}$ & $\begin{array}{c}\text { Binary } \\
\left(b_{0} b_{1} b_{2} b_{3}\right)\end{array}$ & $c_{i}=\left[c_{i, 0} c_{i, 1} c_{i, 2} \cdots c_{i, 30} c_{i, 31}\right]$ \\
\hline 0 & 0000 & 11011001110000110101001000101110 \\
\hline 1 & 0001 & 11101101100111000011010100100010 \\
\hline 2 & 0010 & 00101110110110011100001101010010 \\
\hline 3 & 0011 & 00100010111011011001110000110101 \\
\hline 4 & 0100 & 01010010001011101101100111000011 \\
\hline 5 & 0101 & 00110101001000101110110110011100 \\
\hline 6 & 0110 & 11000011010100100010111011011001 \\
\hline 7 & 0111 & 10011100001101010010001011101101 \\
\hline 8 & 1000 & 10001100100101100000011101111011 \\
\hline 9 & 1001 & 10111000110010010110000001110111 \\
\hline 10 & 1010 & 01111011100011001001011000000111 \\
\hline 11 & 1011 & 01110111101110001100100101100000 \\
\hline 12 & 1100 & 00000111011110111000110010010110 \\
\hline 13 & 1101 & 01100000011101111011100011001001 \\
\hline 14 & 1110 & 10010110000001110111101110001100 \\
\hline 15 & 1111 & 11001001011000000111011110111000 \\
\hline
\end{tabular}

Each data symbol spreads to 32 chip sequences in such a way that the chip rate is 32 times the symbol rate. As shown in Fig. 2, the even numbered indexes $(0,2, \ldots, 30)$ among 32 chip sequences get mapped to I-phases by O-QPSK block while the odd numbered indexes $(1,3, \ldots, 31)$ get mapped to Q-phase. To form the offset between chip modulation between I-phase and Q-phase, the Q-phase chip is delayed by $T_{c}$ compared to I-phase chip.

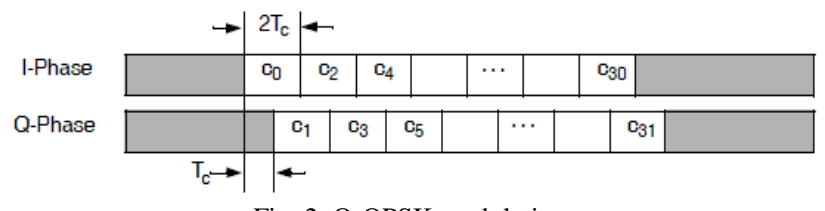

Fig. 2. O-QPSK modulation.

As IEEE 802.15.4 technology allows the error in the frequency in the RF hardware between receiving and sending as wide as $\pm 80 \mathrm{ppm}( \pm 196 \mathrm{KHz})$ [4], it needs an algorithm to remove the error in the frequency of the received signal and detects the signal from the frequency having error for the detection of signals from receivers. Generally, the non-coherent detection method is used as it is resistant to the carrier frequency offset (CFO) [5]. But in this paper the coherent detection method is used in which the carrier frequency offset (CFO) occurring from the nonconformity of oscillator of the sender and receivers is removed and the symbol is detected through correlation to estimate the channel of wireless environment. 


\section{ESTIMATION OF WIRELESS CHANNEL IN HIGH SPEED TRAIN}

\section{A. Carrier Frequency Offset Estimation}

As the IEEE 802.15.4 is a system with a big carrier frequency offset (CFO) of $\pm 80 \mathrm{ppm}$ due to unconformity of the difference in frequency of the oscillator, the CFO should be estimated and compensated prior to the estimation of channels. Generally, it can be modeled as (1) in relation to the CFO.

$$
\hat{y}(n)=x(n) e^{j\left(\Delta \omega n T_{s}\right)}+\hat{w}(n)
$$

where $\hat{y}(n)$ means the received signal containing the component of the CFO, $x(n)$ means the sending signal, $\hat{w}(n)$ means the noise, $\Delta \omega$ means the $\mathrm{CFO}$ and $T_{s}$ means the sampling period.

The most representative estimation method for $\mathrm{CFO}$ is a method of using the correlation between symbols in the range of preamble [6]. In the range of preamble, the symbol value of 0 is repeated 8 times. If we assume that the packets are synchronized, the symbols can be assumed to be $s_{n}=s_{n+M}, \quad i=0,1, \ldots, M-1$ due to the characteristics of the range of preamble. The nth sample should be paired and is multiplied with the $n+M$ th sample. The CFO can be obtained from the following equation (2) and the CFO can be removed using the obtained values.

$$
\begin{aligned}
K & =\sum_{n=0}^{M-1} \hat{y}(n) \hat{y}^{*}(n+M) \\
& =e^{-j \Delta \omega M T_{s}} \sum_{n=0}^{M-1}\left\{|x(n)|^{2}+\hat{w}(n) \hat{w}^{*}(n+M)\right\} \\
\therefore & \Delta \omega=\frac{1}{M T_{s}} \angle K^{*}
\end{aligned}
$$

\section{B. Analysis of the Wireless Channel under High Speed Train}

In the wireless channel environment, the distorted signal is received due to the ISI caused by the multipath component. Its block diagram can be shown as in Fig. 3.

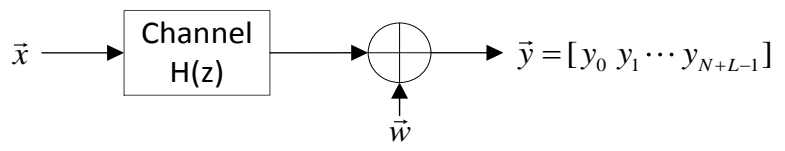

Fig. 3. Channel \& noise block-diagram.

In IEEE 802.15.4 communication, the received signal vector $\vec{y}$ can be expressed in the following form when the sending signal $\vec{x}$ is distorted by the CIR vector $\vec{h}$ and the noise vector $\vec{w}$ containing the white noise and many interference signals is added. At this time, suppose that the $\mathrm{CFO}$ component is removed.

$$
\vec{y}=X \cdot \vec{h}+\vec{w}
$$

where

$$
\begin{gathered}
\vec{y}=\left[\begin{array}{llll}
y_{0} & y_{1} \cdots & y_{N-1}
\end{array}\right]^{T} \\
\vec{h}=\left[\begin{array}{llll}
h_{0} & h_{1} & \cdots & h_{L-1}
\end{array}\right]^{T} \\
\vec{w}=\left[\begin{array}{llll}
w_{0} & w_{1} & \cdots & w_{N-1}
\end{array}\right]^{T} \\
X=\left[\begin{array}{cccc}
x_{0} & x_{-1} & \cdots & x_{-L+1} \\
x_{1} & x_{0} & \cdots & x_{-L+2} \\
\vdots & \vdots & \ddots & \vdots \\
x_{N-1} & x_{L-2} & \cdots & x_{N-L}
\end{array}\right]
\end{gathered}
$$

And, $N$ means the length of the sending signal and $L$ means the length of the channel.

There is the least-square (LS) method as the general channel estimation method using a known-pattern [7]. LS method is the linear method to minimize the difference between the received signal and the sending signal using the channel. Here, the solution of the estimation of the channel through LS methods is given as follows.

$$
\hat{h}_{L S}=\left(X^{*} \cdot X\right)^{-1} X^{*} \vec{y}=\vec{h}+\left(X^{*} \cdot X\right)^{-1} X^{*} \vec{w}
$$

The sequence of estimating the channel using the measured signal is shown in Fig. 4. First, the packet is synchronized and then the channel is estimated using the signal which is an estimate of the CFO and compensation and using LS method.

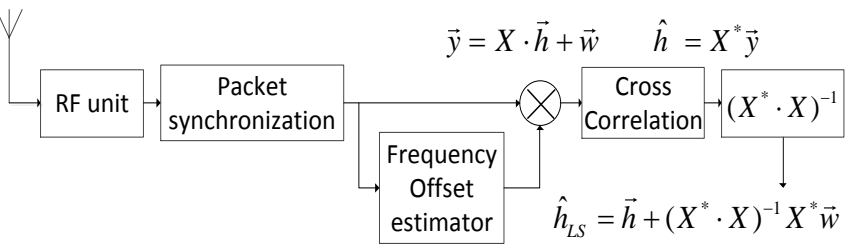

Fig. 4. Least-square(LS) channel estimation.
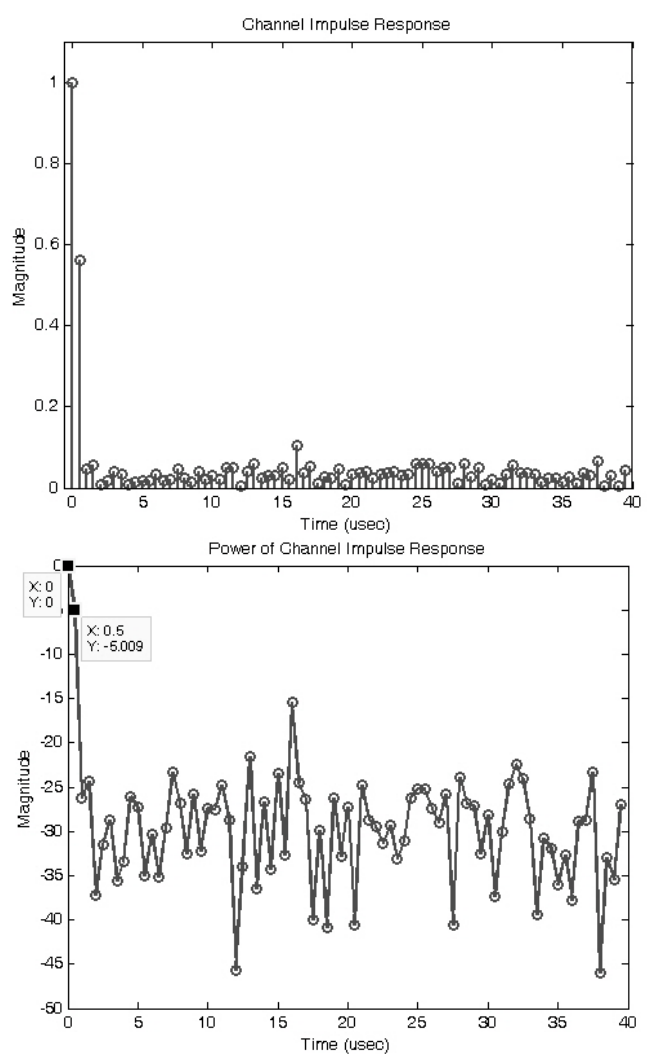

Fig. 5. Analysis result of wireless channel in the train environment. (Top) Linear scale, (Bottom) dB scale. 
Fig. 5 shows the representative case of results of the estimated channel applying LS method to the measured signals. It shows that the size of the first tap is the biggest, followed by the second tap. At this time, if it is normalized using the first tap as a standard, the second tap has the power attenuation of $-5 \mathrm{~dB}$ compared to the first tap. At this time, the distance between taps is the same with the chip period and can be expressed as $T_{c}$.

\section{Proposed Method of Estimation AND DEtECTION}

\section{A. 2-Tap Simple Channel Estimation}

In Chapter III, the channel was analyzed using the measured signals in the communication between the inside and the outside of the high speed train. At this time, as the main components of first tap and second tap have sufficient magnitude and the other taps do not have sufficient magnitude. If the results of channel impulse response(CIR) is applied to 2-tap delay model, the received signal model can be expressed as follows.

$$
\begin{gathered}
y(n)=\sum_{l=0}^{L-1} h(l)\left(x(n) \sin \left(\pi \frac{T_{c}+T_{c} l}{2 T_{c}}\right)+x(n-1) \sin \left(\pi \frac{T_{c} l}{2 T_{c}}\right) j\right) \\
=h(0) x(n)+h(1) x(n-1)
\end{gathered}
$$

where, $x$ means, the received signal, $h$ means the channel tap, $n$ means the index of the received signal and $L$ means the length of channel.

There are 16 data symbols in the band range of $2.45 \mathrm{GHz}$ in the IEEE 802.15.4 standard with each symbol composed of 32-bits chip sequence. The chip sequence of the near symbol is like what is shifted by 4 bits in the cyclic shift and is located in an orthogonal position. If the 2-tap delay model with the length of $4 T_{c}$ between channel taps is used, the chip sequence can be expressed as follows.

$$
C_{m}=\left[\begin{array}{llllllllll}
c_{m, 0} & c_{m, 1} & c_{m, 2} & c_{m, 3} & c_{m, 4} & \cdots & c_{m, 28} & c_{m, 29} & c_{m, 30} & c_{m, 31} \\
c_{m, 28} & c_{m, 29} & c_{m, 30} & c_{m, 31} & c_{m, 0} & \cdots & c_{m, 24} & c_{m, 25} & c_{m, 26} & c_{m, 27}
\end{array}\right]^{T}
$$

At this time, the $C_{m}$ 's auto-correlation matrix is

$$
C_{m}^{H} C_{m}=\left[\begin{array}{cc}
32 & 0 \\
0 & 32
\end{array}\right]
$$

Using this characteristic, the wireless channel can be estimated as follows without the pilot signal.

$$
\hat{h}_{\text {new }}=\frac{1}{32} C_{m}^{H} Y
$$

where, $Y$ means the received signal of 32 chip sequences.

If the method of (6) above is used, the channel $\hat{h}_{\text {new }}$ of tap components which are the first tap and fifth tap is estimated. At this time, the fifth tap is delayed by $4 T_{c}$ compared with the first tap. However, when the channel was analyzed using the measured signals communicated between the inside and the outside of the high speed train, it was checked that the component which is delayed by $T_{c}$ was found, thus obtaining the receiving signal as shown in (5). Now, the matrix of $\hat{C}$ which is delayed by $T_{c}$ as shown above can be expressed as follows.

$$
\hat{C}_{m}=\left[\begin{array}{llllllllll}
c_{m, 0} & c_{m, 1} & c_{m, 2} & c_{m, 3} & c_{m, 4} & \cdots & c_{m, 28} & c_{m, 29} & c_{m, 30} & c_{m, 31} \\
c_{m, 31} & c_{m, 0} & c_{m, 1} & c_{m, 2} & c_{m, 3} & \cdots & c_{m, 27} & c_{m, 28} & c_{m, 29} & c_{m, 30}
\end{array}\right]^{T}
$$

where, the auto-correlation matrix of $\hat{C}_{m}$ is

$$
\hat{C}_{m}^{H} \hat{C}_{m}=\left[\begin{array}{ll}
32 & 4 j \\
4 j & 32
\end{array}\right]
$$

And,

$$
\tilde{h}_{\text {new }}=\frac{1}{32} \hat{C}_{m}^{H} Y
$$

As the first row and second row of $\hat{C}$ are not orthogonal each other, the value $\hat{h}_{\text {new }}$ of may be slightly incorrect. But, the channel $\hat{h}_{\text {new }}$ of tap components can be estimated weakly.

If either of zero forcing (ZF) method and the minimum mean square error (MMSE) method is applied using the estimated channel component through the proposed 2-tap simple channel estimation, the multipath components can be removed.

\section{B. Symbol Detection Method}

The symbol detection method which does not usually consider the channel is the correlation detection using the correlation between received signals and sending signals of chip sequence. It is to find out the highest value by calculating the correlation between chip sequence of all symbols and the received signal. It can be expressed as follows.

$$
\hat{X}(n)=\max _{m}\left\{c_{m}^{H} \cdot Y\right\}
$$

where $c_{m}$ means the chip sequence made of 32 chips which are mapped on the $m$-th symbol and $Y$ means the received signal.

The symbol detection method of (8) is the simplest method using the chip sequence. But as it does not consider the distortion made by the channel, the performance of receiving may be degraded by ISI if there is any multipath delay component in the channel. So, to remove ISI, the above mentioned ZF or MMSE algorithm is applied. But this method has some disadvantages as it needs an inverse matrix, which is hard to obtain.

Accordingly, we proposed the simple symbol detection algorithm considering the multipath delay component. The symbol detection algorithm proposed in this paper is to find out the symbol which has the highest correlation with the received symbol and which can enable the estimated channel value $\tilde{h}_{\text {new }}$ convolute with the chip sequence. It is the method to be applied to both, the removal of ISI and detection of 
symbol for simple detection. It can be expressed as follows.

$$
\hat{X}(n)=\max _{m}\left\{\left(\operatorname{conv}\left(c_{m}, \tilde{h}_{\text {new }}\right)\right)^{H} \cdot Y\right\}
$$

\section{RESUlt AND ANALYSis OF SimULATION}

In this paper, the multipath profile was extracted using the received data measured based on IEEE 802.15.4 communication system in the actual high speed train. Based on the extracted multipath profile, the simple 2-tap channel estimation method and symbol detection method are proposed.

First, the mean square error(MSE) was checked to find out the performance of the proposed channel. The Fig. 6 shows comparison of MSE performance between proposed channel estimation method of (7) and LS channel estimation method when the second multipath delay is $T_{c}$. Although the channel estimation method of (7) may be a little incorrect compare to LS channel estimation method, it can estimate the channel to some extent.

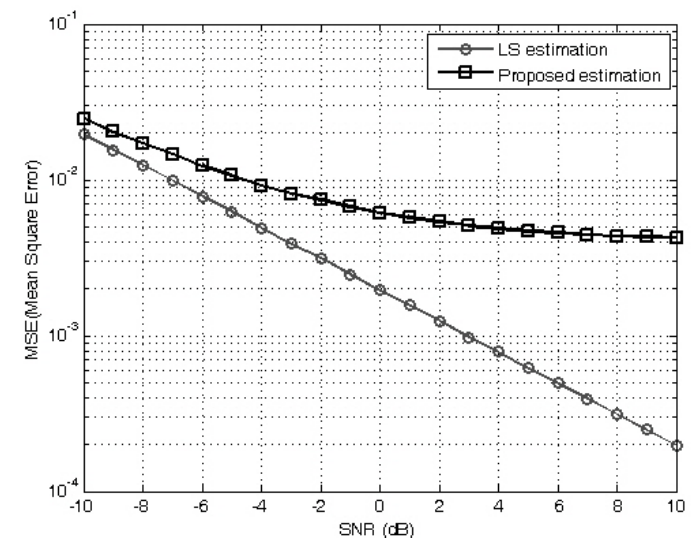

Fig. 6. Mean square error(MSE) of proposed method and LS method.

Second, to evaluate the applicability of $\tilde{h}_{\text {new }}$ by proposed 2-tap simple channel estimation method, the bit error rate(BER) performance simulation was compared by MMSE equalization method according to ideal channel $h$ or estimated channel $\tilde{h}_{\text {new }}$. Assuming that the simulation has the complete synchronization, the timing offset and frequency offset were not considered in the simulation and the applied parameters are summed up in Table III. Especially, the multipath delay \& power parameters are applied with 2-tap AWGN channel model based on the first tap and second tap which have the significant power components as shown in Fig. 5, which were obtained by using the measured signal as specified above.

TABLE III: SIMULATION PARAMETER

\begin{tabular}{cc}
\hline \hline Parameters & Value \\
\hline Chip rate \& sampling rate & $2 \mathrm{MHz}$ \\
Frame structure & Data frame $(802.15 .4)$ \\
Modulation & O-QPSK \\
Spreading Factor & 32 \\
Carrier frequency & $2.45 \mathrm{GHz}$ \\
Packet Size & $50 \mathrm{~K} \mathrm{Bytes}$ \\
Channel & AWGN \& Fast fading channel \\
Delay \& power profile & 0 nsec $(0 \mathrm{~dB}), 500 \mathrm{nsec}(-5 \mathrm{~dB})$ \\
Equalization & MMSE \\
\hline \hline
\end{tabular}

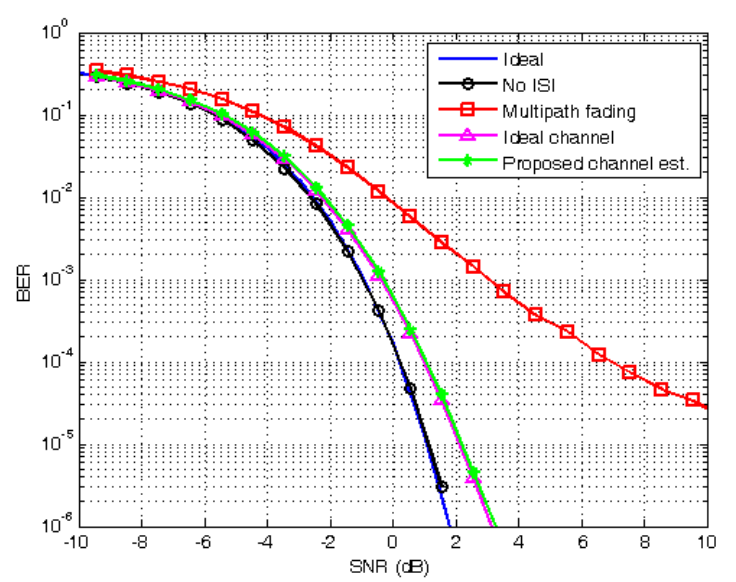

Fig. 7. BER performance by channel estimation methods (Estimation channel by proposed method VS Ideal channel).

The simulation in Fig. 7 shows the result of evaluating the BER performance. The results show that there has been an improvement of performance of more than $4 \mathrm{~dB}$ compared to the state when the equalization method is not applied based on the BER $10^{-3}$. They also show that if the ideal channel $h$ and estimated channel $\tilde{h}_{\text {new }}$ by proposed 2-tap simple channel estimation method of (7) are applied to the general symbol detection method of (8) including MMSE equalization, they show the almost similar performances.

To evaluate the proposed symbol detection method of (9), we compared proposed method to general method as shown in Fig. 8. Next is the simulation result of BER performance when the proposed symbol detection method is compared with the general symbol detection including MMSE equalization. At this time, the channel information was applied through the proposed 2-tap simple channel estimation of (7).

When the proposed symbol detection algorithm is compared to the BER performance of general symbol detection including MMSE equalization, they show almost the same performance with the difference of $0.1 \mathrm{~dB}$. In addition, it shows an excellent BER performance with the difference with ideal BER performance of only $1 \mathrm{~dB}$.

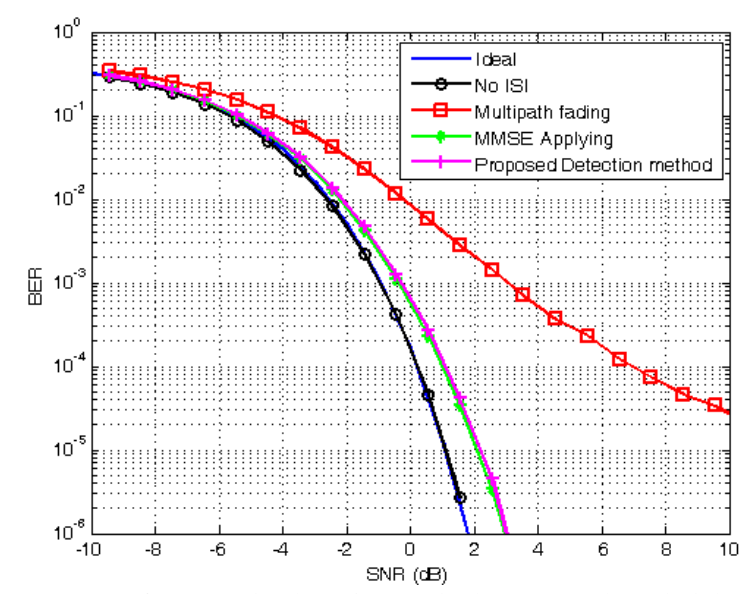

Fig. 8. BER performance by detection methods (Proposed symbol detection method VS MMSE \& correlation method).

\section{CONCLUSION}

In this paper, the multipath components are extracted from the received data measured based on the IEEE 802.15.4 
communication system in the actual high speed train. Then, based on the extracted multipath profile, the simple 2-tap channel estimation method was proposed and it was found out to be excellent through the BER performance simulation. The channel information estimated through the proposed method is less accurate than LS channel estimation method. But, the proposed channel estimation method can be easily applied to the high speed train channel where the 2-tap components are significant using the characteristics of chip sequence. In addition, the proposed symbol detection algorithm can simply remove ISI and detect the symbols and it has almost the same performance when compared with MMSE method. The proposed channel estimation method and the symbol detection method did not show any degradation of the performance compared with general methods in BER performance simulation. So, it is expected that they are the most optimal methods in the train environment where the 2-tap multipath delay component are significant.

\section{ACKNOWLEDGMENT}

The This work was supported by $R \& D$ Convergence Program of MSIP (Ministry of Science, ICT and Future Planning) and ISTK (Korea Research Council for Industrial Science and Technology) of Republic of Korea(Grant B551179-12-06-00) and the C-ITRC(Convergence Information Technology Research Center) support program(NIPA-2014-H0401-14-1002) supervised by the NIPA(National IT Industry Promotion Agency). And, all authors are supported by second stage BK21 project of Korean Research Foundation.

\section{REFERENCES}

[1] J. H. Kim and J. Y. Lee, "A study on the applicability of energy harvesting technology for the sensor network of railroad system by thermal deviation," in Proc. International Conference on Sensor Technologies and Applications 2012, Rome, Italy, Aug. 2012.

[2] Y. S. Kim, K. H. Kim, J. G. Lee, and Y. L. Kim, "Analysis of sensing data for low power transmission under high-speed railway environment," in Proc. International Conference on Advances in Computing, Communications and Informatics 2013, Bangkok, Taiwan, Sept. 2013.

[3] IEEE Standard for Local and Metropolitan Area Networks - Part 15.4: Low-Rate Wireless Personal Area Networks(LR-WPANs), IEEE Std 802.15.4-2011.

[4] A. M. Mehta and K. S. J. Pister, "Frequency offset compensation for crystal-free 802.15.4 communication," Advanced Technologies for Communication(ATC) 2011, Da Nang, Vietnam, Aug. 2011.

[5] S. Farahani, Zigbee Wireless Networks and Transceivers, $1^{\text {st }}$ ed. Elsevier Ltd., 2008, pp. 321-322.

[6] T. Weiss, A. Krohn, and F. Jondral, "Synchronization algorithms and preamble concepts in spectrum pooling systems," IST Mobile and Wireless Telecommunication, Portugal, June 2003.

[7] J. H. Choi, Optimal Combining and Detection, 1st ed. Cambridge University Press, 2010, pp. 49-77.

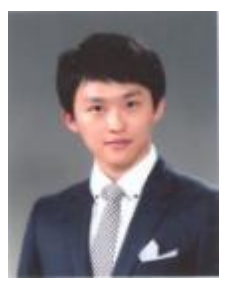

Jingu Lee received his B.S. degree in radio science engineering from Kongju National University, Chungnam-do, Korea in 2013. He is currently working towards the M.S. degree in electronical engineering in Sogang University. His research interests include DSP algorithms, signal processing for communication.

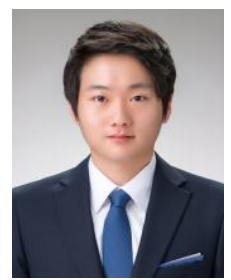

Minho Kim received his B.S. degree in electronic engineering from Chonbuk National University, Jeonju, Korea in 2014. He is currently working towards the M.S. degree in electronical engineering in Sogang University. His research interests include DSP algorithms, signal processing for communication.

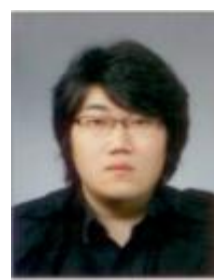

Daehyun Kim received his B.S. degree in electronic engineering from Sogang University, Seoul, Korea in 2014. He is currently working towards the M.S. degree in electronical engineering in Sogang University. His research interests include DSP algorithms, signal processing for communication.

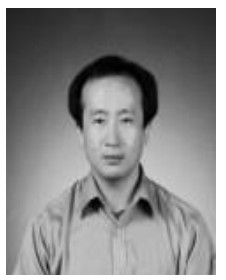

JongHwa Lee received the B.S. and M.S degrees in 1991 and 1993 and is currently pursuing the Ph.D. degree in electronic engineering from Sogang University, Seoul, Korea. From 1995 to 2000, he was a senior engineer with the Hyundai Electronic Industries. From 2000 to 2013, he was a technical director with Telechips Inc. His research interest includes the development of multimedia and communication SoCs, signal processing algorithms.

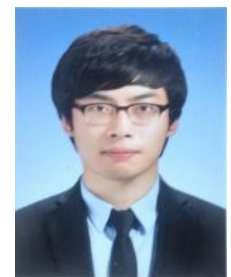

Jungyeol Kim received his B.S. degree in electronic engineering from Sogang University, Seoul, Korea in 2014. He is currently working towards the M.S. degree in electronical engineering in Sogang University. His research interests include DSP algorithms, signal processing for communication.

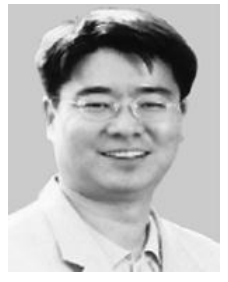

Younglok Kim received his B.S. degree in electronic engineering from Sogang University, Seoul, Korea in 1991 and the M.S. and Ph.D. degrees in electrical engineering from Polytechnic Institute of NYU, Brooklyn, NY in 1993 and 1998 respectively. From 1999 to 2003, he was a senior system engineer at InterDigtal communication Corp. in Melville, NY working on 3GPP wireless systems. Since 2003, he joined the Department of Electronic Engineering in Sogang University, where he is now a professor. His research interests include DSP algorithms, signal processing for communication and radar. 